\title{
Observations of the Pulsating White Dwarf G 185-32
}

\author{
B. G. Castanheira ${ }^{1}$, S. O. Kepler ${ }^{1}$, P. Moskalik ${ }^{2,3}$, S. Zoła ${ }^{3}$, G. Pajdosz ${ }^{3}$, J. Krzesiński ${ }^{3}$, D. O’Donoghue ${ }^{4}$, M. Katz ${ }^{4}$,
}

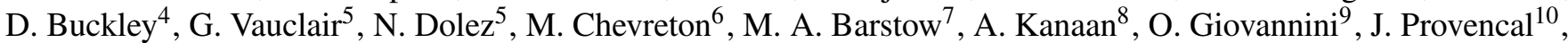
S. D. Kawaler ${ }^{11}$, J. C. Clemens ${ }^{12}$, R. E. Nather ${ }^{13}$, D. E. Winget ${ }^{13}$, T. K. Watson ${ }^{14}$, K. Yanagida ${ }^{13}$, J. S. Dixson ${ }^{13}$, C. J. Hansen ${ }^{15}$, P. A. Bradley ${ }^{16}$, M. A. Wood ${ }^{17}$, D. J. Sullivan ${ }^{18}$, S. J. Kleinman ${ }^{19}$, E. Meišstas ${ }^{20}$, J.-E. Solheim ${ }^{21}$, A. Bruvold ${ }^{21}$, E. Leibowitz ${ }^{22}$, T. Mazeh ${ }^{22}$, D. Koester ${ }^{23}$, and M. H. Montgomery ${ }^{24}$

${ }^{1}$ Instituto de Física, Universidade Federal do Rio Grande do Sul, 91501-900 Porto-Alegre, RS, Brazil

2 Copernicus Astronomical Center, Ul. Bartycka 18, 00-716 Warsaw, Poland

3 Mt. Suhora Observatory, Cracow Pedagogical University, Ul. Podchorazych 2, 30-084 Cracow, Poland

4 South African Astronomical Observatory, PO Box 9, Observatory 7935, SA

${ }^{5}$ Université Paul Sabatier, Observatoire Midi-Pyrénées, CNRS/UMR5572, 14 av. E. Belin, 31400 Toulouse, France

6 Observatoire de Paris-Meudon, DAEC, 92195 Meudon, France

7 Department of Physics and Astronomy, Leicester University, University Road, Leicester, LE1 7RH, UK

8 Departamento de Física, Universidade Federal de Santa Catarina, CP 476, 88040-900, Florianópolis, Brazil

9 Departamento de Física e Química, Universidade de Caxias do Sul, Caxias do Sul, RS - CEP 95001-970, Brazil

10 Department of Physics and Astronomy, University of Delaware, Newark, DE 19716, USA

11 Department of Physics and Astronomy, Iowa State University, Ames, IA 50011, USA

12 Department of Physics, University of North Carolina, Chapel Hill, NC 27599-3255, USA

13 Department of Astronomy and McDonald Observatory, University of Texas, Austin, TX 78712, USA

14 Southwestern University, Georgetown, USA

15 JILA, University of Colorado, Boulder, CO, USA

16 Los Alamos National Laboratory, X-2, MS T-085, Los Alamos, NM 87545, USA

17 Dept. of Physics and Space Sciences \& The SARA Observatory, Florida Institute of Technology, Melbourne, FL 32901, USA

18 Victoria University of Wellington, PO Box 600, Wellington, New Zealand

19 Sloan Digital Sky Survey, Apache Pt. Observatory, PO Box 59, Sunspot, NM 88349, USA

20 Institute of Theoretical Physics and Astronomy, Gostauto 12, Vilnius 2600, Lithuania

${ }^{21}$ Institutt for Fysikk, Universitetet i Troms $\varnothing$, 9037 Troms $\emptyset$, Norway

22 Wise Observatory, Tel Aviv University, Tel Aviv 69978, Israel

23 Institut für Theoretische Physik und Astrophysik, Universität Kiel, Germany

24 Institute of Astronomy, Madingley Road, Cambridge, CB3 OHA, UK

Received 6 June 2003 / Accepted 23 September 2003

\begin{abstract}
We observed the pulsating hydrogen atmosphere white dwarf G 185-32 with the Whole Earth Telescope in 1992. We report on a weighted Fourier transform of the data detecting 18 periodicities in its light curve. Using the Hubble Space Telescope Faint Object Spectrograph time resolved spectroscopy, and the wavelength dependence of the relative amplitudes, we identify the spherical harmonic degree $(\ell)$ for 14 pulsation signals. We also compare the determinations of effective temperature and surface gravity using the excited modes and atmospheric methods, obtaining $T_{\text {eff }}=11960 \pm 80 \mathrm{~K}, \log g=8.02 \pm 0.04$ and $M=0.617 \pm 0.024 M_{\odot}$.
\end{abstract}

Key words. stars: white dwarfs - stars: variables: general - stars: oscillations - stars: individual: G 185-32 - stars: evolution

\section{Introduction}

G 185-32, also called PY Vul and WD1935+279, is a pulsating white dwarf with a hydrogen atmosphere, i.e., a DAV. It was discovered to pulsate by McGraw et al. (1981), who

Send offprint requests to: B. G. Castanheira, e-mail: barbara@if.ufrgs.br found a complex period structure of small amplitude, with the main periodicity at $P=215 \mathrm{~s}\left(f_{0}\right)$, and others at $141 \mathrm{~s}\left(3 f_{0} / 2\right)$ and $71 \mathrm{~s}\left(3 f_{0}\right)$.

Kepler et al. (2000) studied G 185-32 Hubble Space Telescope (HST) Faint Object Camera time series spectra, and detected periodicities at $70.9 \mathrm{~s}, 72.5 \mathrm{~s}, 141.8 \mathrm{~s}, 215.7 \mathrm{~s}, 300.0 \mathrm{~s}$, $301.3 \mathrm{~s}, 370.1 \mathrm{~s}$ and $560.0 \mathrm{~s}$. They show that the amplitude of 
the periodicity at $141.8 \mathrm{~s}$ does not increase toward the ultraviolet as predicted by $g$-mode pulsation models (Robinson et al. 1982; Kepler 1984; Robinson et al. 1995).

Among all pulsating white dwarfs, this star has the shortest periodicity so far observed. However, the peak-to-peak amplitude is small in comparison with other ZZ Ceti of similar periods. The star shows short timescale periodicities, e.g. $215 \mathrm{~s}$, as well as long ones, e.g. $560 \mathrm{~s}$ (Kepler et al. 2000). The stars at the blue edge (hot DAVs) of the ZZ Ceti instability strip present few, short period and low amplitude periodicities. On the other hand, the stars at the red edge (cool DAVs) have many periodicities, with their high amplitude periodicities being longer than 600 s (see e.g. Fig. 1 in Kanaan et al. 2002).

Previous work on atmospheric parameter determinations was undertaken by Bergeron et al. (1995); they obtained $\log g=8.05 \pm 0.05$ and $T_{\text {eff }}=12130 \pm 200 \mathrm{~K}$ for ML2 $/ \alpha=0.6$ model atmosphere fit to the optical spectra. Koester \& Allard (2000) used the observed $V$ magnitude, parallax and UV spectrum to obtain $\log g=7.92 \pm 0.10$ and $T_{\text {eff }}=11820 \pm 110 \mathrm{~K}$.

Thompson \& Clemens (2003) reported time resolved spectroscopy obtained using a Keck telescope and proposed that the pulsation axis has an inclination of $90 \mathrm{deg}$ to the lineof-sight, as they did not observe any velocity variations. The Keck data was taken on two nights, for $3.5 \mathrm{hr}$ each night, so the time resolution is low. Central to their interpretation was a small peak at $285.1 \mathrm{~s}$ (their $f_{3}$ ). They suggested this as a normal mode at $285.1 \mathrm{~s}$ with peaks at $141.9 \mathrm{~s}\left(2 f_{3}\right), 95.1 \mathrm{~s}\left(3 f_{3}\right)$, and $70.9 \mathrm{~s}\left(4 f_{3}\right)$ as harmonics.

\section{Observations}

We observed ${ }^{1}$ G 185-32 with the Whole Earth Telescope (WET) in 1992, during the eighth WET run (Xcov8), as shown in Table $1^{2}$.

During Xcov8, the WET operated telescopes at eight sites located around the globe (see Table 1); all eight telescopes operated with various designs of two star photometers (Nather et al. 1990), and collected a total of $76.4 \mathrm{hr}$ worth of data, for a duty cycle of $34 \%$. As the data spans $226 \mathrm{hr}$, the resolution was $1.2 \mu \mathrm{Hz}$. The second channel of the photometer was monitoring a nearby star to assure that variations on the light curve were not due to variable sky transparency.

The runs were reduced and analysed as described by Kepler (1993): the total light curve is a simple combination of the light curves obtained at each telescope, after reducing all data to normalized modulation (fractional) intensities, and times in relation to the barycenter of the solar system, the uniform Barycentric Coordinate Time (BCT) scale (Standish 1998). The Fourier transform of the reduced and time corrected light curve obtained with the WET is displayed in Fig. 1.

\footnotetext{
${ }^{1}$ Partially based on observations at Observatório do Pico dos Dias, operated by Laboratório Nacional de Astrofísica, MCT, Brazil, and Cerro Tololo Interamerican Observatory, operated by NOAO for the NSF.

2 The WET is a collaboration of astronomers to observe variable stars with periods of a few minutes, typical of pulsating white dwarf stars (Nather et al. 1990).
}

Table 1. Journal of observations

\begin{tabular}{lllrr}
\hline \hline Telescope & Run & Date & UT & Length \\
& & & & $(\mathrm{s})$ \\
\hline Suhora 0.6 m & x8004 & 1992 Sep. 21 & $21: 55: 45$ & 1540 \\
Suhora 0.6 m & x8005 & 1992 Sep. 21 & $22: 21: 50$ & 6650 \\
LNA 1.6 m & ro017 & 1992 Sep. 22 & $0: 04: 20$ & 8810 \\
McDonald 2.1 m & pab-0142 & 1992 Sep. 22 & $3: 33: 30$ & 10770 \\
Suhora 0.6 m & x8007 & 1992 Sep. 22 & $19: 12: 20$ & 13185 \\
LNA 1.6 m & ro019 & 1992 Sep. 22 & $22: 20: 10$ & 14430 \\
Suhora 0.6 m & x8009 & 1992 Sep. 23 & $19: 05: 00$ & 15185 \\
McDonald 2.1 m & pab-0146 & 1992 Sep. 24 & $1: 47: 00$ & 18210 \\
Suhora 0.6 m & x8010 & 1992 Sep. 24 & $18: 58: 00$ & 13760 \\
McDonald 2.1 m & pab-0152 & 1992 Sep. 25 & $1: 46: 30$ & 14590 \\
Mauna Kea 0.6 m & maw-0103 & 1992 Sep. 25 & $5: 47: 15$ & 8130 \\
Suhora 0.6 m & x8012 & 1992 Sep. 25 & $18: 08: 45$ & 18335 \\
Suhora 0.6 m & x8014 & 1992 Sep. 26 & $17: 31: 25$ & 14060 \\
Suhora 0.6 m & x8015 & 1992 Sep. 26 & $21: 32: 15$ & 9570 \\
La Palma INT 2.5 m & int-0018 & 1992 Sep. 26 & $22: 33: 00$ & 11765 \\
McDonald 2.1 m & pab-0159 & 1992 Sep. 27 & $1: 46: 00$ & 21870 \\
Mauna Kea 0.6 m & maw-0106 & 1992 Sep. 27 & $7: 17: 30$ & 7875 \\
Suhora 0.6 m & x8017 & 1992 Sep. 27 & $18: 04: 25$ & 10205 \\
La Palma INT 2.5 m & int-0021 & 1992 Sep. 27 & $20: 02: 00$ & 18800 \\
CTIO 1.5 m & jlp-0125 & 1992 Sep. 27 & $23: 57: 30$ & 7175 \\
McDonald 2.1 m & pab-0162 & 1992 Sep. 28 & $1: 48: 00$ & 17220 \\
Mauna Kea 0.6 m & maw-0108 & 1992 Sep. 28 & $5: 17: 00$ & 14845 \\
Siding Spring 1.0 m & sjk-0204 & 1992 Sep. 28 & $9: 54: 00$ & 12260 \\
Maidanak 1.0 m & jesem-14 & 1992 Sep. 28 & $15: 58: 20$ & 11035 \\
McDonald 2.1 m & pab-0165 & 1992 Sep. 29 & $1: 45: 30$ & 20285 \\
Mauna Kea 0.6 m & maw-0109 & 1992 Sep. 29 & $5: 13: 20$ & 19445 \\
Siding Spring 1.0 m & sjk-0205 & 1992 Sep. 30 & $9: 33: 00$ & 5300 \\
Mauna Kea 0.6 m & maw-0113 & 1992 Oct. 01 & $5: 20: 00$ & 10865 \\
Mauna Kea 0.6 m & maw-0116 & 1992 Oct. 03 & $5: 21: 00$ & 1070 \\
\hline & & & & \\
\hline
\end{tabular}

In order to have some objective criterion for determining which peaks are real in the discrete Fourier transform, we adopt here an amplitude limit such that a peak exceeding this limit has only $1 / 1000$ probability of being due to noise (false alarm probability). Kepler (1993) and Schwarzenberg-Czerny (1991, 1999), following Scargle (1982), demonstrated that nonequally spaced data sets, like WET data sets, do not have a normal noise distribution, because the residuals are correlated. In this case, peaks above $4\langle A\rangle$ ( 4 times the square root of the average power), have probability 1 in 1000 of being noise. In addition to the periods found by Kepler et al. (2000), we also found the following periodicities in the WET data set: $651.6 \mathrm{~s}$, $266.1 \mathrm{~s}, 264.2 \mathrm{~s}, 212.8 \mathrm{~s}, 148.5 \mathrm{~s}, 141.2 \mathrm{~s}$, and $72.9 \mathrm{~s}$. We did not find the periodicity at $560 \mathrm{~s}$ in the WET data, which appears in the HST Fourier spectrum reported by Kepler et al. (2000).

To know if a peak in the Fourier transform is a periodicity intrinsic or only due to the spectral window, we subtracted, from the light curve, the sine curve with the same amplitude 

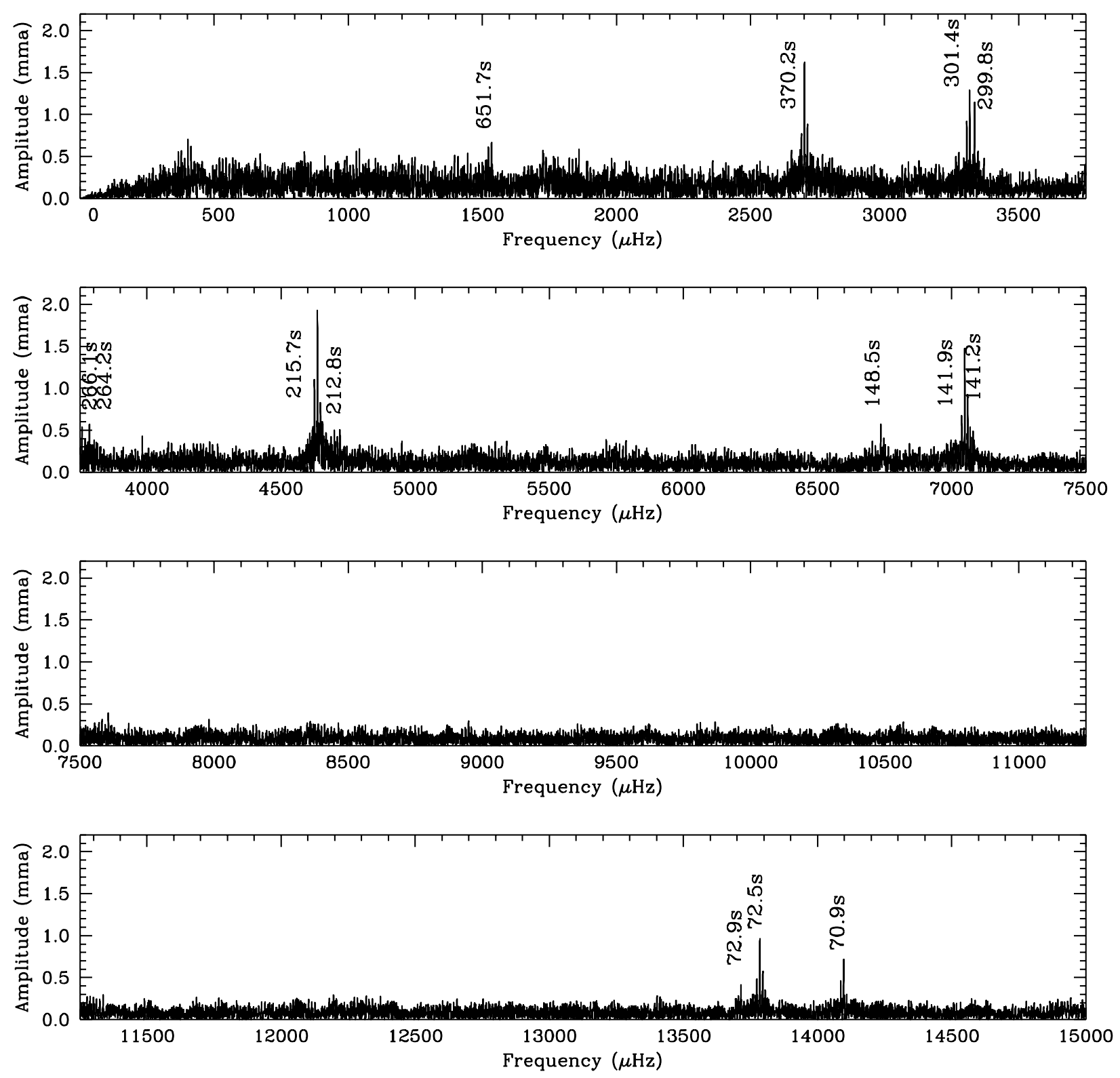

Fig. 1. Fourier transform of the total WET (Xcov8) data set. The periodicities are listed in Table 2.

and period as the peak we selected in the Fourier transform, including its phase information. After subtracting it from the light curve, we re-calculated the Fourier transform to verify if the sine curve was correct. Then, we repeat the procedure for the remaining periodicities.

In the Fourier transform presented in Fig. 1, we did not consider any weighting due to telescope aperture, observation site or data length. We discuss our weighting scheme for the WET data and the results of our analysis in the next section.

\section{Fourier transform with weights}

To improve the signal-to-noise ratio, we calculated a weighted Fourier transform; the weights depend not only on the telescope size and the number of data points acquired, but also on the weather conditions and peculiarities of the site and instrument.
Handler (2003) explores different weighting schemes and concludes the best choice is the inverse of the scatter.

Kepler (1993) demonstrated that the noise in a Fourier transform can be estimated from the average amplitude in the frequency range of interest, the square root of the average power. Our procedure to estimate the weights was first to subtract from each individual light curve all the periodicities detected in the Fourier transform above four times the average amplitude, i.e., with a probability of being due to noise (false alarm probability) smaller than $1 / 1000$. This guarantees that the average amplitude calculated is not affected by the presence of the large amplitude pulsation modes.

After the subtraction, we calculated the average amplitude of each individual run, thus estimating its noise. We define the weight as the inverse of the average amplitude squared, because the noise level in the Fourier transform is smaller than if we had considered the weight as the inverse of the average amplitude 


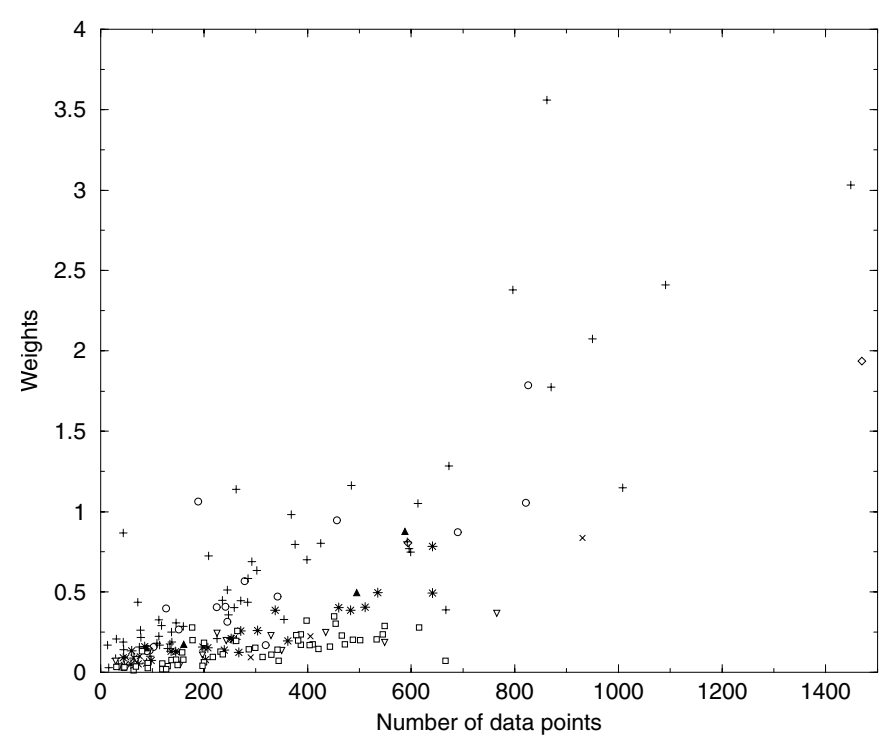

Fig. 2. The weights are the inverse of the average amplitude squared, calculated from the Fourier transform of each chunk, after subtracting all periodicities above $4\langle A\rangle$ in the WET Fourier transform, from each light curve. The different telescopes are represent by: La Palma $2.5 \mathrm{~m}$ (open circle), Maidanak $1.0 \mathrm{~m}$ (diamond), Tololo $1.5 \mathrm{~m}$ (filled triangle up), Mauna Kea $0.6 \mathrm{~m}$ (star), McDonald $2.1 \mathrm{~m}$ (plus), LNA $1.6 \mathrm{~m}$ (open triangle down), Siding Spring $1 \mathrm{~m}(\mathrm{x})$ and Suhora $0.6 \mathrm{~m}$ (open square). Each run was separated into chunks, if there were interruptions longer than $35 \mathrm{~s}$. The data with small weights contribute relatively little to the weighted Fourier transform.

as suggested by Handler (2003). In Fig. 2, we show the weights calculated for each chunk of data, using different telescopes. Even for the same telescope and night (run), the weather conditions are critical in determining the noise. The weighted Fourier transform of the WET data is displayed in Fig. 3. With this approach, we identified two further periodicities in the light curve: $537.6 \mathrm{~s}$ and $454.6 \mathrm{~s}$.

Figure 4 is a comparison between the spectral window for a given mode, with and without weights. The spectral window is the Fourier transform of a single coherent frequency in a light curve with the same gaps, sampling and total exposure time as the original data. As we are applying various weights to different sections of the overall light curve, some segments will have small weighting, which is equivalent to using less data. The spectral window of the data with weights has therefore lower resolution, but the signal-to-noise ratio in the weighted Fourier transform is higher. The change in the measured noise, represented by the average amplitude $\langle A\rangle$, is an estimate of the improvement: it changes from 0.154 to 0.140 when we move from no-weights to weights, i.e., a $10 \%$ improvement.

\section{The HST data set}

As described by Kepler et al. (2000), the HST data set consists of series of $10 \mathrm{~s}$ exposures in the range 1180-2508 $\AA$, and a zeroth-order simultaneous observation with an effective wavelength at $3400 \AA$, which has a counting rate around 100 times larger than the UV data. As the HST data cover a total of $15.2 \mathrm{hr}$, its time resolution, around $18 \mu \mathrm{Hz}$, is low compared to that of the WET data, around $1.2 \mu \mathrm{Hz}$. We therefore used the frequencies of periodicities detected with the WET, and the periodicities only detected in the Fourier transform of the HST data, to carry out simultaneous multisinusoidal nonlinear least squares fit to the zeroth-order HST data, assuming that the excited pulsations, when present, have the same frequency, as is the case for the DAV G 29-38 (Kleinman et al. 1998) and for the DBV GD 358 (Kepler et al. 2003). We do not use any amplitude from WET data on the HST data analysis, just the frequencies.

Using a randomization (Monte Carlo simulation) of the HST data, as described by Kepler (1993), we determined that the $1 / 1000$ probability of a peak being due to noise in the HST Fourier transform occurs around 3.3 $\langle A\rangle$. Figure 5 shows the Fourier transform of the zeroth-order data and the 1/1000 false alarm probability line. We detected in the HST data periodicities at $264 \mathrm{~s}, 266 \mathrm{~s}$ and $182 \mathrm{~s}$, which also appear in WET Fourier transform above $2.3\langle A\rangle, 3.0\langle A\rangle$ and $1.5\langle A\rangle$, respectively. We also detected the periodicity at $148 \mathrm{~s}$ which was also detected in WET data set. These four periodicities were not reported by Kepler et al. (2000).

We list, in Table 2, all periodicities detected in the WET data set and in our analysis of the HST data set. The listed amplitudes, the phases and their uncertainties, were obtained by a simultaneous multisinusoidal least squares fit to the WET and HST data sets. We forced the WET and HST data to fit all these periodicities. The times of maxima $\left(T_{\max }\right)$ for the HST data are given in relation to $T_{0}=$ 2449929.9333442 BCT, while the WET data are given in relation to $T_{0}=2448887.416559 \mathrm{BCT}$. Our frequency uncertainties do not allow bridging the $3 \mathrm{yr}$ data gap.

The exposures with the Faint Object Spectrograph (FOS) of the HST used the blue Digicon detector and the G160L grating, and consist of 764 useful pixels over the spectral region 1150 to $2510 \AA$, each with a width of $1.74 \AA$ per pixel. The UV photometry, reported as HST 1180-2508 $\AA$, in Table 2, was obtained just adding the spectra over all wavelengths.

We can measure reliable amplitudes only for bins redder than approximately $1200 \AA$ because of contamination of the observed spectra by geocoronal emission. To increase the signal-to-noise ratio, we convolved the theoretical amplitude spectra into $50 \AA$ A bins, obtaining amplitudes directly comparable to normalized binned measurements.

We then proceeded with a simultaneous multisinusoidal least squares fit to the different binned wavelength light curves, calculating amplitudes and phases for the detected periodicities in all wavelength. Robinson et al. (1982) demonstrate that the phases for $g$-modes in white dwarf models are the same at all wavelengths, when there are no significant nonadiabatic effects. Figure 6 shows that, for the main periodicity at $215 \mathrm{~s}$, the phase does not change with wavelength.

We also detected in the HST data a periodicity around $45 \mathrm{~min}$, which is caused by the movement of the star in the aperture caused by the wobbling of the HST solar panels when they come in and out of the shadow of the Earth. We included this periodicity in our multisinusoidal fit, to reduce the uncertainties. 

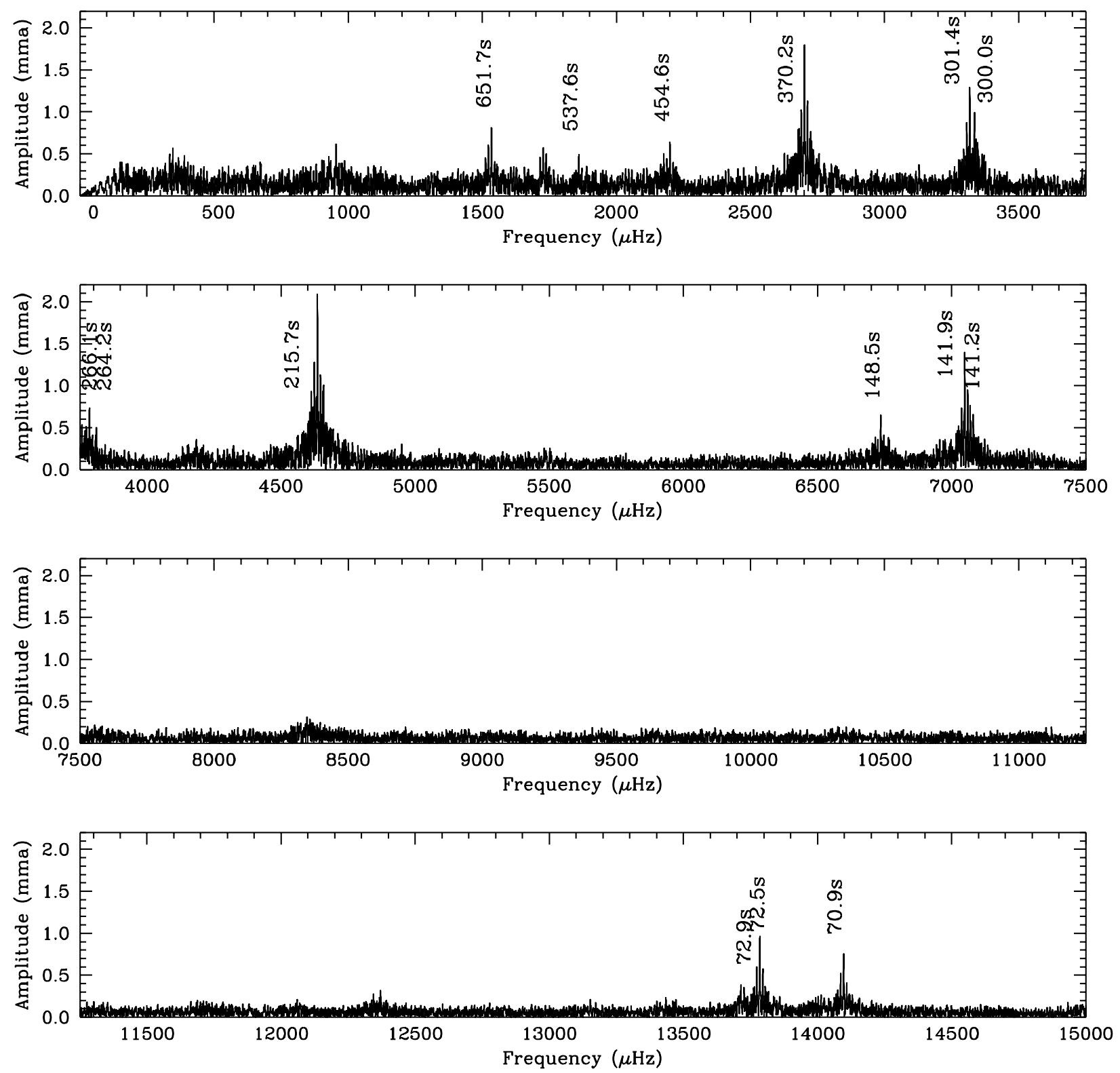

Fig. 3. Fourier transform of the total WET data with weights. Periodicities detected are listed in Table 2 . The periodicity around $1730 \mu \mathrm{Hz}$ is not present in the HST data set and is bellow our detection limit.

We looked for possible linear combination modes as in most of the pulsating white dwarf stars with a large number of periodicities detected, a substantial fraction of these frequencies can be attributed to linear combinations and harmonics of a smaller number of parent modes (e.g. Kepler et al. 2003). This does not appear to be the case for G 185-32. Table 3 lists the possible linear combinations detected, considering the obtained amplitudes from the WET data. On the other hand, considering the amplitudes from HST, and therefore in the UV, where most of the emission is, we must write the linear combination frequencies as $f_{651 \mathrm{~s}}=f_{301 \mathrm{~s}}-f_{560 \mathrm{~s}}$ and $f_{141.9 \mathrm{~s}}=0.5 \times f_{70.9 \mathrm{~s}}$, because the amplitude ratios for these periodicities are the opposite in the UV. The amplitudes of both the harmonics and the linear combination frequencies (e.g., Wu 2001) generated by the nonlinear processes should be smaller than the amplitudes of the parent modes; in G 185-32, the periodicities have similar amplitudes in the optical.

\section{Comparison with theoretical amplitudes}

We used the detected periodicities, and their change in amplitude with wavelength, to calculate the effective temperature $\left(T_{\text {eff }}\right)$, the surface gravity $(\log g)$, and the spherical harmonic degree $(\ell)$ of each pulsation.

We compared the observational changes in amplitude with wavelength to those predicted by the $g$-mode pulsation models described by Robinson et al. (1995) and Kepler et al. (2000), calculated from Koester's model atmospheres, described in Finley et al. (1997). These amplitude calculations take into account the wavelength dependence of the limb darkening and 
Table 2. Periodicities detected in the HST and the WET data sets. The question mark (?) is due to the fact that these periodicities are marginally detected in the WET and HST data sets. The uncertainties for the frequencies detected in the WET data were calculated using nonlinear least squares and are around $1.2 \mu \mathrm{Hz}$. The frequencies detected only in the WET data are $651.7 \mathrm{~s}, 537.6 \mathrm{~s}, 454.6 \mathrm{~s}, 212.8 \mathrm{~s}, 141.2 \mathrm{~s}$ and $72.9 \mathrm{~s}$, and only in the HST data are $181.9 \mathrm{~s}$ and $560.8 \mathrm{~s}$. All the frequencies were included in the linear fit, even if they were not resolved.

\begin{tabular}{|c|c|c|c|c|c|c|c|}
\hline \multirow{2}{*}{$\begin{array}{c}\text { Frequency } \\
(\mu \mathrm{Hz})\end{array}$} & \multirow{2}{*}{$\begin{array}{c}\text { Periods } \\
(\mathrm{s})\end{array}$} & \multirow{2}{*}{$\begin{array}{c}\text { WET } \\
\text { Amplitude } \\
\text { (mma) }\end{array}$} & \multicolumn{2}{|r|}{ HST $3400 \AA$} & \multicolumn{3}{|c|}{ HST 1180-2508 } \\
\hline & & & $\begin{array}{r}T_{\max } \\
(\mathrm{s})\end{array}$ & $\begin{array}{c}\text { Amplitude } \\
\text { (mma) }\end{array}$ & $\begin{array}{r}T_{\max } \\
\text { (s) }\end{array}$ & $\begin{array}{c}\text { Amplitude } \\
\text { (mma) }\end{array}$ & $\begin{array}{r}T_{\max } \\
(\mathrm{s})\end{array}$ \\
\hline 1534.5 & 651.70 & $0.67 \pm 0.07$ & $74.1 \pm 10.8$ & $0.91 \pm 0.12$ & $94.4 \pm 13.6$ & $2.9 \pm 0.3$ & $122.2 \pm 9.7$ \\
\hline 1783.3 & 560.77 & $0.09 \pm 0.07$ & $69.0 \pm 71.0$ & $1.49 \pm 0.12$ & $547.1 \pm 7.2$ & $2.9 \pm 0.3$ & $552.2 \pm 8.3$ \\
\hline $1860.2(?)$ & 537.59 & $0.57 \pm 0.07$ & $132.9 \pm 10.4$ & $0.67 \pm 0.12$ & $67.6 \pm 15.4$ & $1.6 \pm 0.3$ & $67.2 \pm 14.6$ \\
\hline 2199.9(?) & 454.56 & $0.38 \pm 0.07$ & $35.5 \pm 13.1$ & $0.60 \pm 0.12$ & $164.3 \pm 14.5$ & $1.1 \pm 0.3$ & $192.3 \pm 19.2$ \\
\hline 2701.2 & 370.21 & $1.62 \pm 0.07$ & $92.0 \pm 2.5$ & $2.21 \pm 0.12$ & $90.0 \pm 3.2$ & $4.8 \pm 0.3$ & $96.1 \pm 3.3$ \\
\hline 3317.8 & 301.41 & $1.13 \pm 0.07$ & $26.2 \pm 3.0$ & $2.03 \pm 0.12$ & $297.8 \pm 2.8$ & $4.5 \pm 0.3$ & $301.2 \pm 2.9$ \\
\hline 3335.6 & 299.79 & $0.95 \pm 0.07$ & $203.6 \pm 3.5$ & $1.77 \pm 0.12$ & $212.6 \pm 3.2$ & $3.9 \pm 0.3$ & $211.3 \pm 3.3$ \\
\hline 3757.3 & 266.15 & $0.40 \pm 0.07$ & $13.7 \pm 7.5$ & $0.58 \pm 0.12$ & $34.6 \pm 8.9$ & $1.3 \pm 0.3$ & $31.0 \pm 8.8$ \\
\hline 3785.2 & 264.19 & $0.51 \pm 0.07$ & $140.3 \pm 5.8$ & $0.69 \pm 0.12$ & $108.3 \pm 7.4$ & $1.8 \pm 0.3$ & $104.9 \pm 6.5$ \\
\hline 4635.3 & 215.74 & $1.93 \pm 0.07$ & $111.3 \pm 1.2$ & $2.59 \pm 0.12$ & $60.0 \pm 1.6$ & $7.1 \pm 0.3$ & $58.7 \pm 1.3$ \\
\hline 4698.8 & 212.82 & $0.53 \pm 0.07$ & $44.7 \pm 4.4$ & $0.66 \pm 0.12$ & $141.7 \pm 6.1$ & $0.3 \pm 0.3$ & $141.1 \pm 30.5$ \\
\hline 5497.7 & 181.90 & $0.03 \pm 0.07$ & $180.3 \pm 72.2$ & $0.43 \pm 0.12$ & $39.4 \pm 8.0$ & $1.1 \pm 0.3$ & $45.4 \pm 6.9$ \\
\hline 6736.1 & 148.45 & $0.57 \pm 0.07$ & $23.8 \pm 2.9$ & $0.53 \pm 0.12$ & $94.9 \pm 5.4$ & $0.7 \pm 0.3$ & $92.9 \pm 9.2$ \\
\hline 7048.8 & 141.87 & $1.43 \pm 0.07$ & $39.2 \pm 1.1$ & $1.67 \pm 0.12$ & $97.1 \pm 1.6$ & $2.1 \pm 0.3$ & $97.6 \pm 3.0$ \\
\hline 7080.4 & 141.24 & $0.39 \pm 0.07$ & $66.7 \pm 4.0$ & $0.24 \pm 0.12$ & $96.5 \pm 11.4$ & $0.1 \pm 0.3$ & $34.6 \pm 80.0$ \\
\hline 13714.4 & 72.92 & $0.36 \pm 0.07$ & $15.1 \pm 2.3$ & $0.36 \pm 0.12$ & $38.3 \pm 3.9$ & $0.5 \pm 0.3$ & $33.2 \pm 6.0$ \\
\hline 13784.9 & 72.54 & $0.93 \pm 0.07$ & $28.5 \pm 0.9$ & $1.23 \pm 0.12$ & $23.2 \pm 1.1$ & $3.0 \pm 0.3$ & $25.2 \pm 1.0$ \\
\hline 14097.7 & 70.93 & $0.69 \pm 0.07$ & $26.9 \pm 1.1$ & $1.82 \pm 0.12$ & $24.0 \pm 0.7$ & $4.3 \pm 0.3$ & $24.4 \pm 0.7$ \\
\hline
\end{tabular}

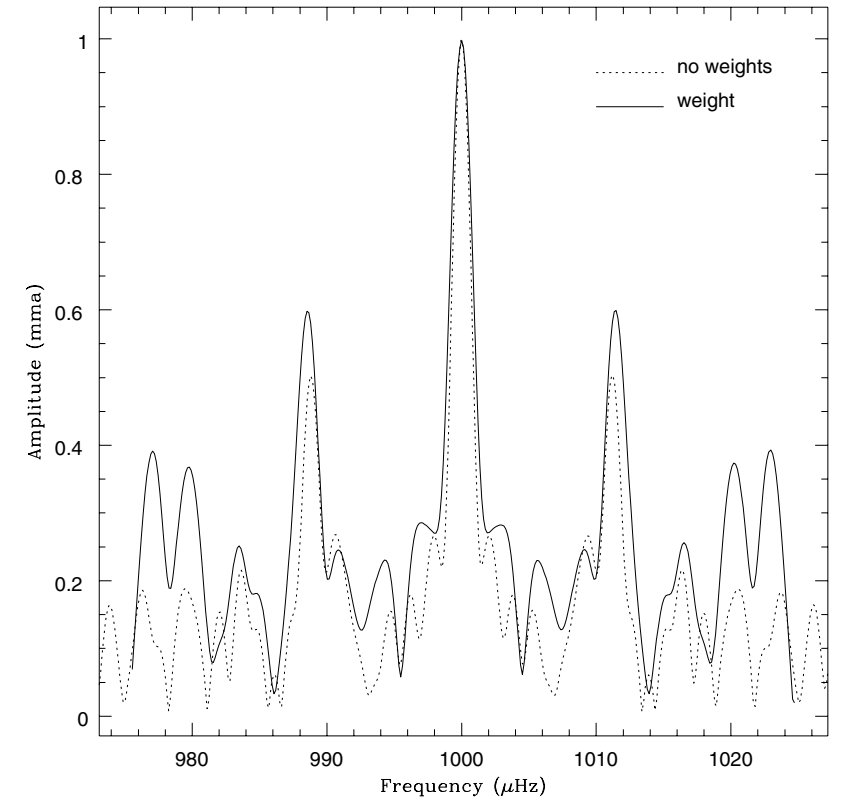

Fig. 4. Spectral Window of the WET data with weighting according to the inverse of the noise squared (solid line) and no weights (dashed line).

different cancellation of the flux variation for different spherical harmonic degrees. Even though Ising \& Koester (2001) show that the effect of the convective zone introduces nonlinearities, amplitude and inclination angle dependence in $A(\lambda)$ are negligible for small amplitudes. It was not possible to include all periodicities listed in Table 2 in the fit because some peaks are not detected in HST data. In addition, as the HST data have low time resolution $(18 \mu \mathrm{Hz})$, nearby periodicities interfere with each other if their frequency difference is smaller than $\Delta f<$ $1 / T$, where $T$ is the total time base of the observation.

By fitting $A(\lambda) / A(3400 \AA)$ to those predicted by the models, which are $\ell$ dependent, we determined $T_{\text {eff }}, \log g$ and $\ell$ for each periodicity, keeping initially these three parameters free. The obvious constraint is that the star must have the same value for $T_{\text {eff }}$ and $\log g$, for all pulsation modes; the $\ell$ value can be different for each pulsational mode. Kepler et al. (2000) determined $\ell$ for the main periodicities, using a fixed $T_{\text {eff }}$ and $\log g$, calculated by other methods.

As each periodicity gives a different value for $T_{\text {eff }}$ and $\log g$, we calculated the local minima, which are the possible solutions in the difference between the observed amplitude versus wavelength curve and the models (predicted amplitudes), i.e., the $\chi^{2}$ of the fit for each periodicity. Using a normal distribution, we estimated probability densities of that local minimum fit. Because we do not know the $\ell$ values for each periodicity, their probability must be added, i.e., the probability for each $\left(T_{\text {eff }}, \log g\right)$ model is the sum of the probabilities for $\ell=1$, 2 and 3. Each one has its effective temperature and surface gravity. Higher values of $\ell$ were discarded because of the extremely high geometrical cancellation in the optical (Robinson et al. 1982) and the absence of phase shift to the UV (Kepler et al. 2000). For each periodicity, we summed all probability densities resulting from local minima. By multiplying all the sums for the different periodicities, we obtain the most probable value of $T_{\text {eff }}=12030_{-200}^{+80} \mathrm{~K}$ and $\log g=8.02_{-0.19}^{+0.07}$. The probability distribution derived by amplitude vs. wavelength, 


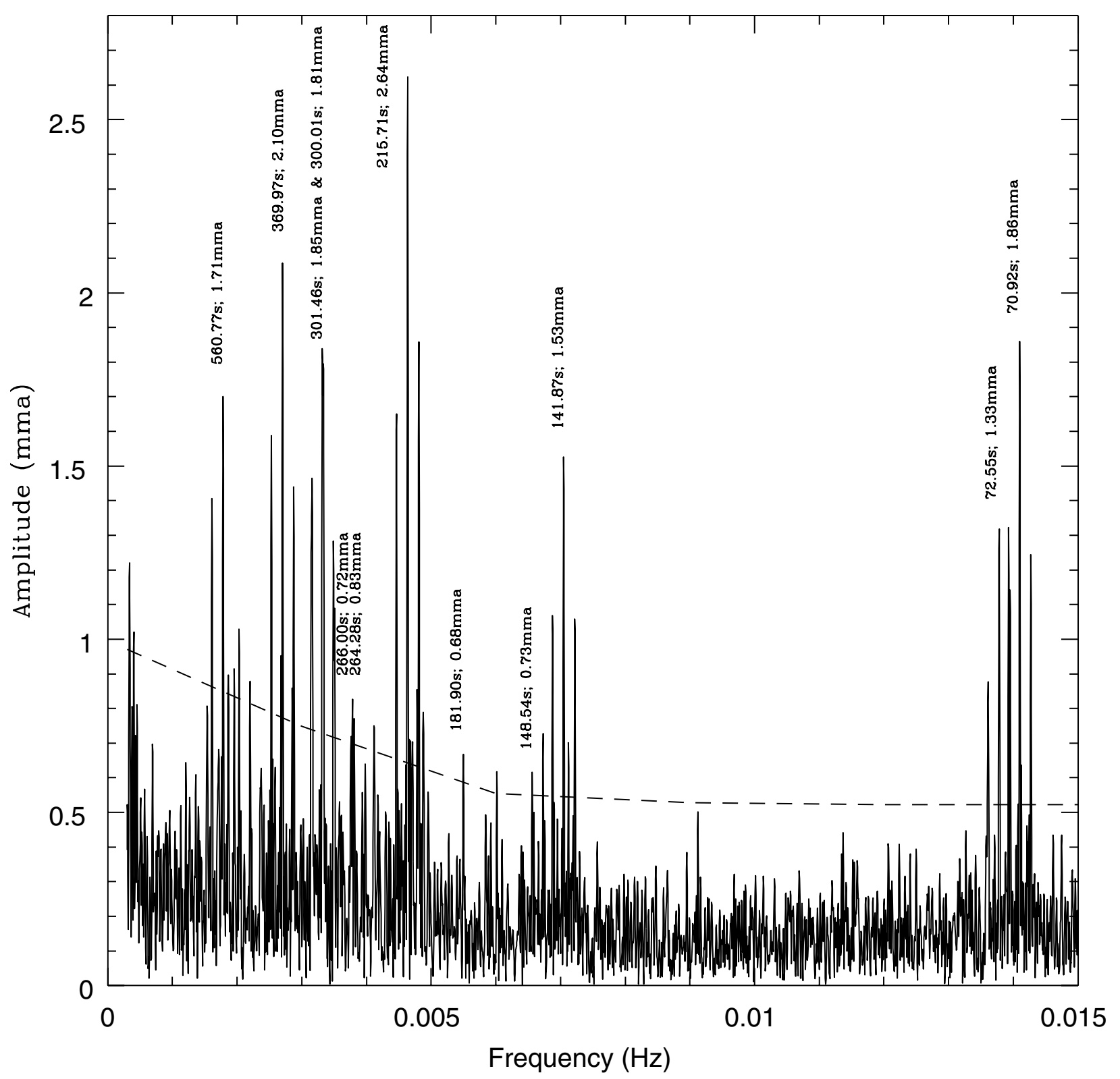

Fig. 5. HST Fourier transform (solid line) and detection limit line above $3.3\langle A\rangle$ (dashed line).

or $A(\lambda)$ variation, is shown in Fig. 7, where we can identify lower probability combined solutions for $T_{\text {eff }}$ and $\log g$ at $(12470,8.23),(12773,8.49)$ and $(13268,8.72)$. Even though the two lower probability solutions are out of the theoretical and observational instability strip, we did not exclude them a priori. For the most probable fit, the best $\ell$ values for each mode are given in Table 4 . The only possibilities are $\ell=1$ or 2 .

\section{The $141.9 \mathrm{~s}$ periodicity}

In Fig. 8 we show how normalized amplitudes change with wavelength for periodicities at $215.7 \mathrm{~s}, 141.9 \mathrm{~s}$ and $70.9 \mathrm{~s}$. It is important to notice that the periodicity at $141.9 \mathrm{~s}$ does not change its amplitude significantly with wavelength, as the others do (same result as Kepler et al. 2000). When we consider that this periodicity does not fit any model and the fact that in
Table 3. Possible linear combination of periodicities detected in G $185-32 .|\Delta f|=\left|f_{\text {obs }}-f_{\text {comb }}\right|$ is $0.08,0.16$ and 0.04 for (a), (b) and (c), respectively.

\begin{tabular}{cccc}
\hline \hline Period & $f_{\text {obs }}$ & Combination & $f_{\text {comb }}$ \\
\hline 560.77 & 1783.25 & $f_{301.4 \mathrm{~s}}-f_{651.7 \mathrm{~s}}$ & $1783.33(\mathrm{a})$ \\
148.45 & 6736.12 & $f_{72.5 \mathrm{~s}}-f_{141.9 \mathrm{~s}}$ & $6736.28(\mathrm{~b})$ \\
70.93 & 14097.70 & $2 \times f_{141.9 \mathrm{~s}}$ & $14097.66(\mathrm{c})$ \\
\hline
\end{tabular}

the UV, where the maximum of the flux distribution occurs, the amplitude of the $141.9 \mathrm{~s}$ periodicity is much smaller than the others, especially when compared to the $71 \mathrm{~s}$ periodicity, we conclude that it is not a $g$-mode pulsation and that it is probably a peak caused by large amplitude effects, i.e., a nonlinear effect. Its period is twice that of the 71 s periodicity. In Fig. 9, 


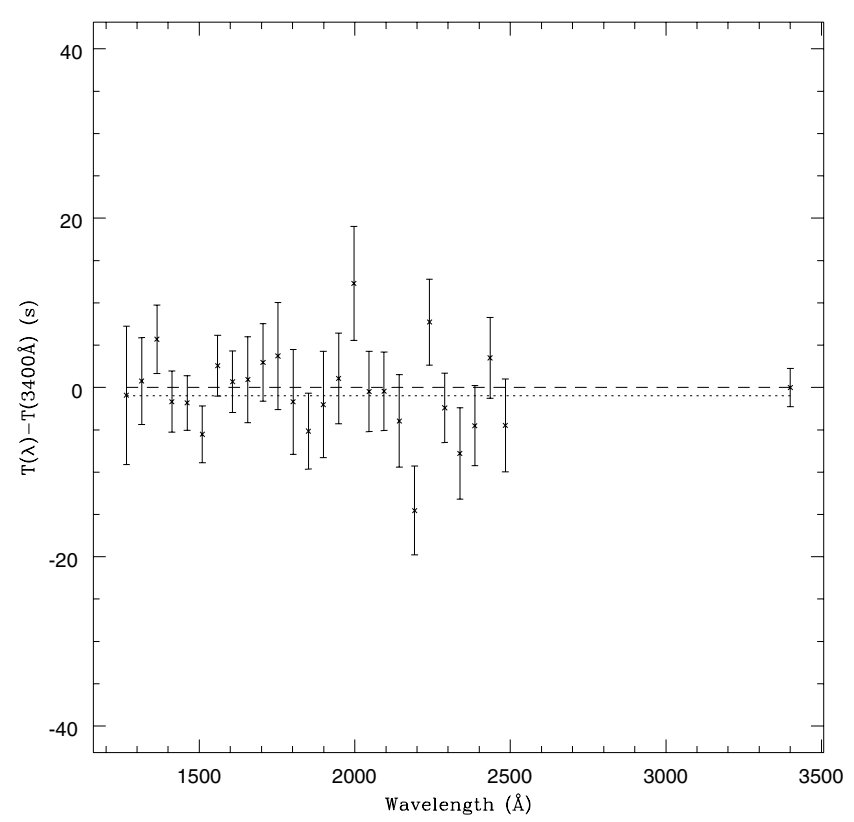

Fig. 6. Phase difference for $P=215 \mathrm{~s}$, where $T(\lambda)$ is the time of maximum at that $\lambda$. The $y$-axis corresponds to $\pm 20 \%$ of one cycle. The dotted line corresponds to the weighted mean of the differences. The dashed line corresponds to the theoretical prediction with no significant nonadiabatic effects, i.e., phases do not change with wavelength. The plotted error bars are 2 sigma total and the uncertainties on the difference are propagated.

Table 4. $\ell$ determination for the most probable model, with $T_{\text {eff }}=$ $12030 \mathrm{~K}$ and $\log g=8.02$. The only possibilities are $\ell=1$ or 2 ; the $\ell=1$ could be 2 with smaller probability and vice-versa.

\begin{tabular}{ll}
\hline \hline Period (s) & $\ell$ \\
\hline 651 & 2 \\
560 & 1 \\
454 & 1 \\
370 & 1 \\
301 & 1 \\
300 & 1 \\
266 & 2 \\
264 & 2 \\
215 & 2 \\
212 & 1 \\
181 & 1 \\
148 & 1 \\
72 & 2 \\
70 & 2 \\
\hline
\end{tabular}

we show that its phase does not change significantly with wavelength, although the uncertainties are significant.

Assuming that we have detected nonlinear effects in the light curve, the intrinsic pulsation amplitudes should be higher than the ones we are detecting in the Fourier transform, because peaks with this amplitude do not normally show significant nonlinear combination peaks. Therefore, the inclination of the pulsation axis to the line of sight must be unfavorable (Pesnell 1985), $90 \mathrm{deg}$ if $m=0$ or $m= \pm 2$ and $0 \mathrm{deg}$ if $m= \pm 1$. The $m=0$ mode propagates from pole to pole and $m= \pm 1$ propagate along the equatorial line. As we also detected periodicities longer than $500 \mathrm{~s}$, typical of cool pulsators, we conclude that the star is not at the blue edge, but actually it is closer to the middle of the instability strip.

\section{The 71 s periodicity}

Another noteworthy periodicity is the one at $70.9 \mathrm{~s}$, the shortest one detected in any pulsating white dwarf. As the $\ell$ value for this periodicity is 2 or 1 , we must analyse all possibilities. Periodicities below $100 \mathrm{~s}$ are predicted by pulsation models for $\ell=1$ and $k=1$, but only if the stellar mass is around $\sim 1.0 M_{\odot}$ or more (Bradley 1996, 2001). Our pulsation analysis and all previous works suggest that the mass of G 18532 is around 0.6 to $0.7 M_{\odot}$. Another possibility would be that the 71 s periodicity were $\ell=1$ and $k=0$; in this case, the center of mass moves during pulsation, which implies that G 185-32 must have a companion. Saffer et al. (1998) searched for an evidence of spectroscopic binarity around several white dwarfs and found none for G 185-32. The star is also not a known proper motion pair. Calculating the semi-major axis that a planet with negligible mass should have for an orbital period of $70.9 \mathrm{~s}$, we found a value of about $20000 \mathrm{~km}$, or about twice the white dwarf radius. This is well inside the Roche limit, and such a planet would not survive, and we can discard this hypothesis.

A much simpler model is obtained if $\ell=2$, which is in agreement with model predictions for a normal mass white dwarf, and consistent with our determination. We examined evolution/pulsation models similar to those described by Bradley $(1996,2001)$ from 0.60 to $0.70 M_{\odot}$, first to see if models that agree with the $\log g$ values can match the periods predicted by seismological models. We then used the observed periods to attempt to constrain the structure of the pulsation models, especially the hydrogen layer mass.

Models with masses between 0.60 to $0.70 M_{\odot}$ constrain the $70.9 \mathrm{~s}$ mode to be the $k=1$ mode if it is $\ell=2$; if this mode were $\ell=1$, the mass would have to be much higher. Identifying the $70.9 \mathrm{~s}$ mode as the $\ell=2, k=1$ mode offers a strong constraint on the hydrogen layer mass as a function of stellar mass. At $0.70 M_{\odot}$, the hydrogen layer mass can be as thin as $1.0 \times 10^{-4} M_{\star}$, while at $0.60 M_{\odot}$, even a hydrogen layer mass of $2.5 \times 10^{-4} M_{\star}$ has an $\ell=2, k=1$ mode period of $75 \mathrm{~s}$. A hydrogen layer this thick on a $0.60 M_{\odot}$ white dwarf is probably not realistic, as $p p$ burning at the base of the hydrogen layer would make the hydrogen layer thinner than this. A $0.65 M_{\odot}$ white dwarf can match the $70.9 \mathrm{~s}$ period with hydrogen layer masses of 1.0 to $1.5 \times 10^{-4} M_{\star}$.

\section{Discussion}

The model proposed by Thompson \& Clemens (2003) attempts to explain why the $141 \mathrm{~s}$ peak does not rise in amplitude towards the ultraviolet while the $71 \mathrm{~s}$ peak does, and also explain non-detection of any velocity variations. If their $285 \mathrm{~s}\left(f_{3}\right)$ peak is the fundamental mode and the pulsation inclination angle were close to 90 deg to the line-of-sight, then the geometrical 


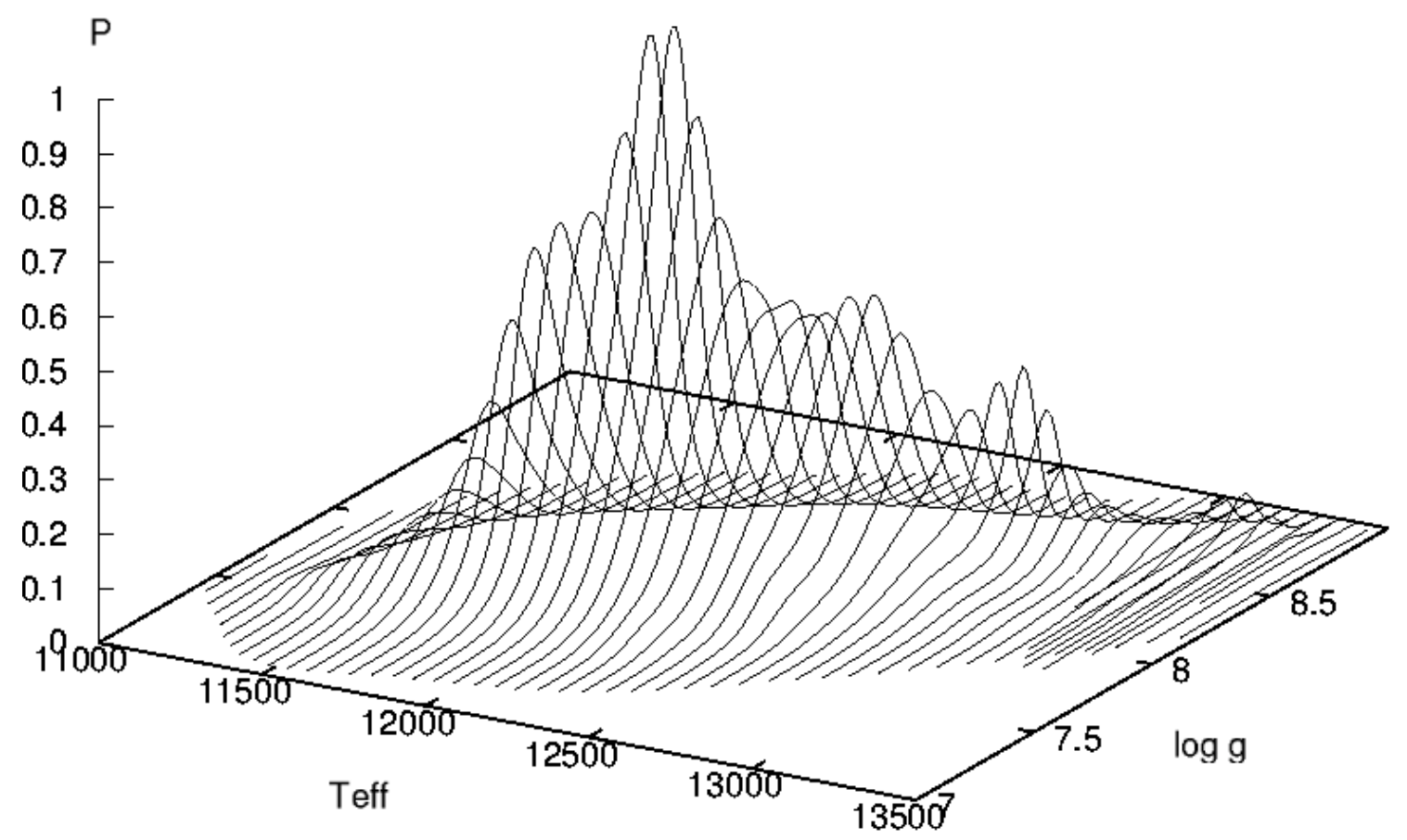

Fig. 7. Product of probability densities of bivariate normal distributions determined from each periodicities. The best solution is $T_{\text {eff }}=12030$ and $\log g=8.02 \mathrm{~K}(P=1) . T_{\text {eff }}$ is the effective temperature, $g$ is the surface gravity, and $P$ is the probability density. The lower probability peaks are at $(12470,8.23)(P=0.5),(12773,8.49)(P=0.34)$ and $(13268,8.72)(P=0.09)$.

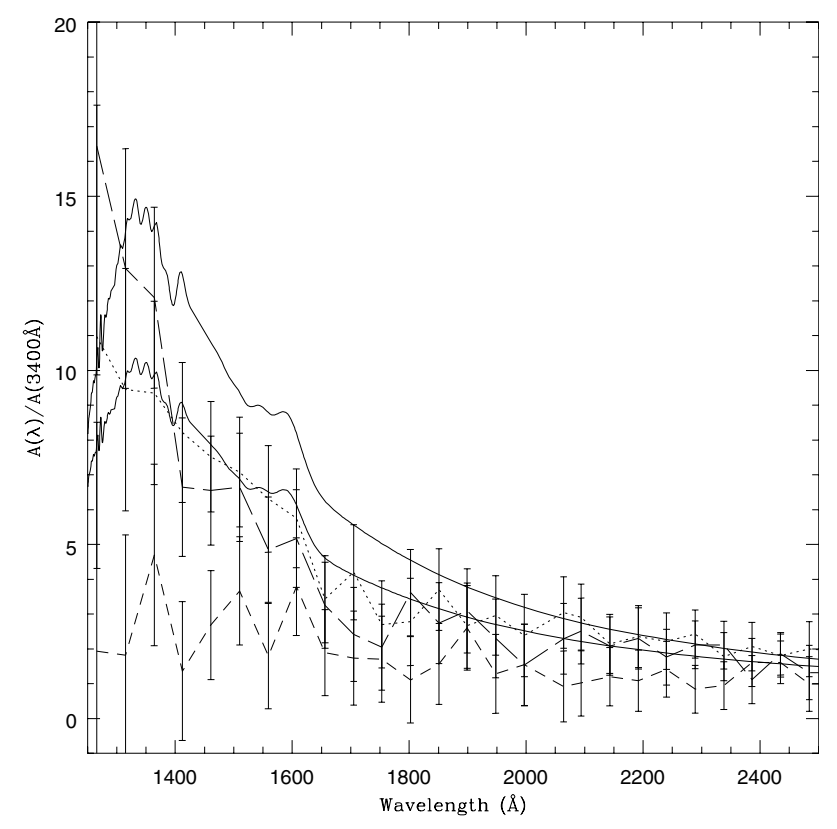

Fig. 8. Amplitude versus wavelength for periodicities at $215 \mathrm{~s}$ (green doted line), $141.9 \mathrm{~s}$ (red short-dashed line) and 70.9s (blue longdashed line). The black solid lines are the models with $T_{\text {eff }}=12000 \mathrm{~K}$ and $\log g=8.0$ with $\ell=2$ (top line) and $\ell=1$ (bottom line). The plotted error bars are 2 sigma total and the uncertainties on the ratios had their uncertainties propagated.

cancellation from the inclination of the pulsation axis could be the answer.

Their proposed value for $f_{3}$ is based on the hypothesis that the $141.9 \mathrm{~s}$ (their value) periodicity is actually the harmonic, $2 f_{3}$. Considering we resolved a peak at $141.87 \mathrm{~s}$ and

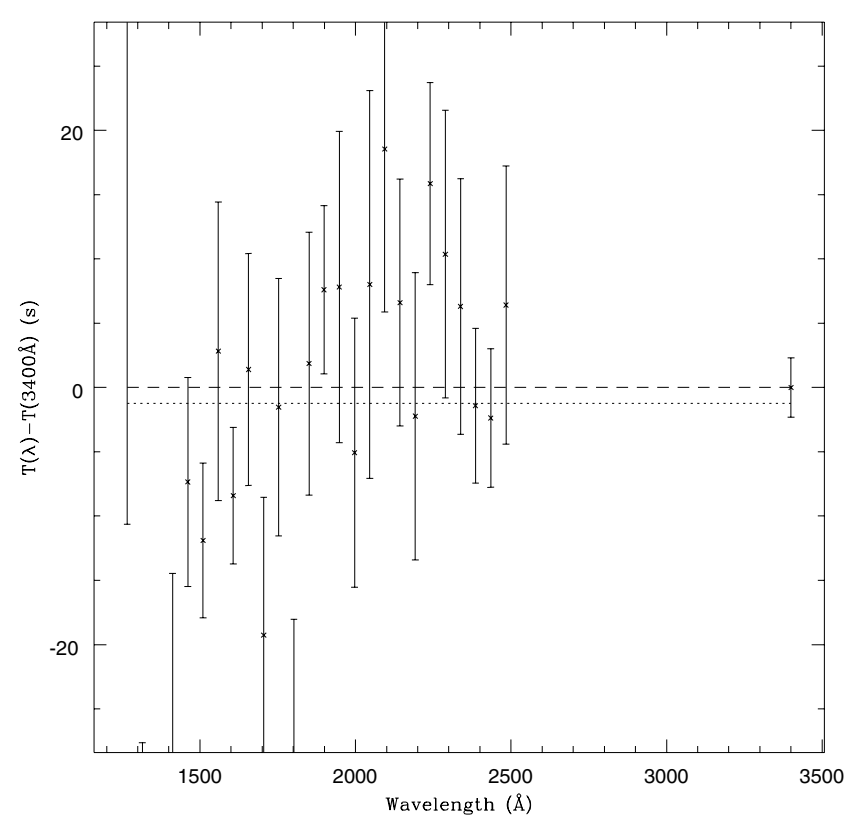

Fig. 9. Phase difference in relation to the phase at $3400 \AA$, for $P=$ $141.9 \mathrm{~s}$, where $T(\lambda)$ is the time of maximum at that $\lambda$. The $y$-axis corresponds to $\pm 20 \%$ of one cycle. The dotted line corresponds to the weighted mean of the differences. The dashed line corresponds to the theoretical prediction with no significant nonadiabatic effects, i.e., phases do not change with wavelength. The plotted error bars are 2 sigma total and the uncertainties on the difference are propagated.

another smaller one at $141.24 \mathrm{~s}$ in the WET data set (see Table 2), we estimated the maximum amplitude for $f_{3}$ in three cases. First, we calculated it by nonlinear least squares fit with simultaneous sinusoids one for each detected peaks described 
in Table 2, assuming our largest periodicity at $141.87 \mathrm{~s}$ were the harmonic of $f_{3}$. Second, we considered the possibility the harmonic was the $m=0$ mode and that we observe the harmonics of the $m=-1$ (at $141.87 \mathrm{~s}$ ) and $m=1$ (at 141.24 s) modes. Third, we used Thompson \& Clemens (2002) published periods. In all cases, there is no detectable pulsation at their $f_{3}$ $(\sim 285 \mathrm{~s})$ or its second harmonic $3 f_{3}(\sim 95 \mathrm{~s})$ in the WET data.

Our upper limits are around $0.14 \mathrm{mma}$, almost the same as our estimate of the noise level, $\langle A\rangle$. The detection limit in the data by Thompson \& Clemens (2003) was $0.17 \mathrm{mma}$.

The measured amplitudes during the WET observation in 1992, with an effective wavelength of $4100 \AA$, are $30 \%$ smaller than the corresponding periodicities at $3400 \AA$ HST data obtained in 1995, while the theoretical models predict only a $3 \%$ decrease due to change in wavelength (Robinson et al. 1982). We note that the published Fourier transform on the discovery runs have larger amplitudes (e.g. $2.8 \mathrm{mma}$ and $2.6 \mathrm{mma}$ for the $215 \mathrm{~s}$ peak). It is therefore clear that the amplitudes change with time, and it is conceivable that the $285.1 \mathrm{~s}$ small amplitude peak detected by Thompson \& Clemens (2003) disappeared in both in the WET and HST observations. However, the Keck data they obtained is low time resolution, and we note that the $285.1 \mathrm{~s}$ periodicity is close to the sidelobes of the periodicities at $301 \mathrm{~s}$ and $300 \mathrm{~s}$ in their data. Note that the beating of unresolved pulsation could also be the cause of the apparent amplitude variation.

Considering the amplitude of the 141.9 s mode does not increase towards the ultraviolet, but the amplitude of the $70.9 \mathrm{~s}$ period does, we propose that the $70.9 \mathrm{~s}$ periodicity is, in fact, a real eigenfrequency of the star, i.e., a real mode. Buchler et al. (1997) show that if there is a resonance between pulsation modes, even if the mode is stable, its amplitude will be necessarily nonzero. Wu \& Goldreich (2002) discuss parametric instability mechanisms for the amplitude of the pulsation modes, but they only discuss the case where the parent mode is unstable and the daughter modes are stable. Even if the observably-large amplitude of the $141.9 \mathrm{~s}$ periodicity were the result of a resonance with a harmonic frequency of another mode, it would still be a mode and its amplitude would depend on wavelength like any other mode. The resonance condition would allow energy to be pumped into the mode, and hence drive it to an observable amplitude, but this resonance mechanism does not change the geometry of the pulsation mode, it only affects the amplitude.

In Table 2, we see other periodicities like $212.8 \mathrm{~s}, 141.2 \mathrm{~s}$ and $72.9 \mathrm{~s}$ that apparently do not change amplitude significantly with wavelength. These periodicities are not resolved in HST data, and as we detected other periodicities close to them, their amplitudes are unreliable.

G 185-32 is a hot DAV, both in terms of its main periodicity being around $215 \mathrm{~s}$ and in terms of its measured effective temperature. Bergeron et al. (1995) defined the instability strip from 12460 to $11160 \mathrm{~K}$ in effective temperature, using ML2 $/ \alpha=0.6$. Their $T_{\text {eff }}=12130 \pm 200 \mathrm{~K}$ fit to the optical spectra of G 185-32 places the star 300 degrees cooler than the blue edge. Koester \& Allard's (2000) determination from the UV shows that the star is also 300 degrees lower than their blue edge, and their instability strip is around 1000 degrees wide. For these reasons, we conclude that the star is not at the blue edge. The calculations of convective driving given in $\mathrm{Wu}$ (1998) and Goldreich \& Wu (1999) were done in the linear limits and estimated the nonlinearities in the light curves as the lowest-order nonlinear corrections (Wu 2001). Given the highly nonlinear sensitivity of the depth of the convection zone to the instantaneous effective temperature, these first-order nonlinear corrections may not accurately reflect the actual nonlinearities observed in a large amplitude pulsator. In fact, some pulsators have large enough amplitudes and are close enough to the blue edge of the instability strip that their convection zones should essentially disappear during the temperature maximum in a pulsation cycle. This does not mean, however, that the convection zone cannot produce driving or nonlinearities, as during temperature minimum the depth and therefore the heat capacity of the convection zone will be increased and will be large enough to modulate the flux. Thus, while the depth of the convection zone may be too small to produce driving or nonlinearities, over the entire pulsation cycle, a significant amount of driving and flux modulation (nonlinearities) can still result. It is important to notice that even if the $141.9 \mathrm{~s}$ periodicity were represented by $Y_{\ell, m}^{2}$ effects, it can be decomposed into a sum of spherical harmonics. In fact, $Y_{1, m}^{2}=\frac{1}{\sqrt{4 \pi}} Y_{0, m}+\frac{1}{\sqrt{5 \pi}} Y_{2, m}$, so we would expect that the wavelength dependence of its amplitude to be between that of an $\ell=0$ and an $\ell=2$ mode. The result is the same if we choose any $m$ value. $Y_{1, m}^{2}$ is the first approximation on a Taylor series expansion, consistent with a treatment of nonlinear effects as a perturbation. On the other hand, if the modes we detect at $141.9 \mathrm{~s}$ and $141.2 \mathrm{~s}$ were the result of the $m$-degeneracy removal (eg. due to rotation), than the cancellation caused by stronger limb darkening in the ultraviolet should not be as effective as that seen in the observations. In Fig. 10 we show the amplitude versus wavelength for the periodicity at $141.9 \mathrm{~s}$, in comparison with models with $Y_{1, m}^{2}, \ell=0, \ell=1$ and $\ell=2$, for a model with $T_{\text {eff }}=12000 \mathrm{~K}$ and $\log g=8.00$. We note that observations are closer to $Y_{1, m}^{2}$, but the data do not fit it. We emphasize that this periodicity is not $\ell=0$ (radial mode), as its period should be less than $3 \mathrm{~s}$ (Robinson 1979). The $3400 \AA$ data is simultaneous with the UV data, in spite of the data not resolving the $141.2 \mathrm{~s}$, this effect is cancelled out when we divide the amplitudes $A(\lambda)$ by $A(3400 \AA)$.

The fact that the amplitude of $141.9 \mathrm{~s}$ does not change with wavelength indicates that this periodicity does not correspond to an actual pulsation mode, but is most likely the result of nonlinear effects. The major difference between the $141.9 \mathrm{~s}$ mode of G185-32 and nonlinear combination peaks of other ZZ Ceti stars is that the $141.9 \mathrm{~s}$ mode is a "difference" mode and is large amplitude. We have not seen this before, and the theory to explain this is not in place yet.

As the observed amplitudes are low compared to other ZZ Cetis of same periods (e.g. Kanaan et al. 2002), we agree that the pulsation axis is probably close to perpendicular to the line-of-sight $\left(i \approx 0^{\circ}\right)$, as suggested by Thompson \& Clemens (2003, TC), even though the $m= \pm 1$ modes, if present, will not cancel out as the $m=0$ modes (or vice-versa). The fact that TC did not detect any velocity variation requires that only the $m=0$ modes be excited throughout all the pulsation 


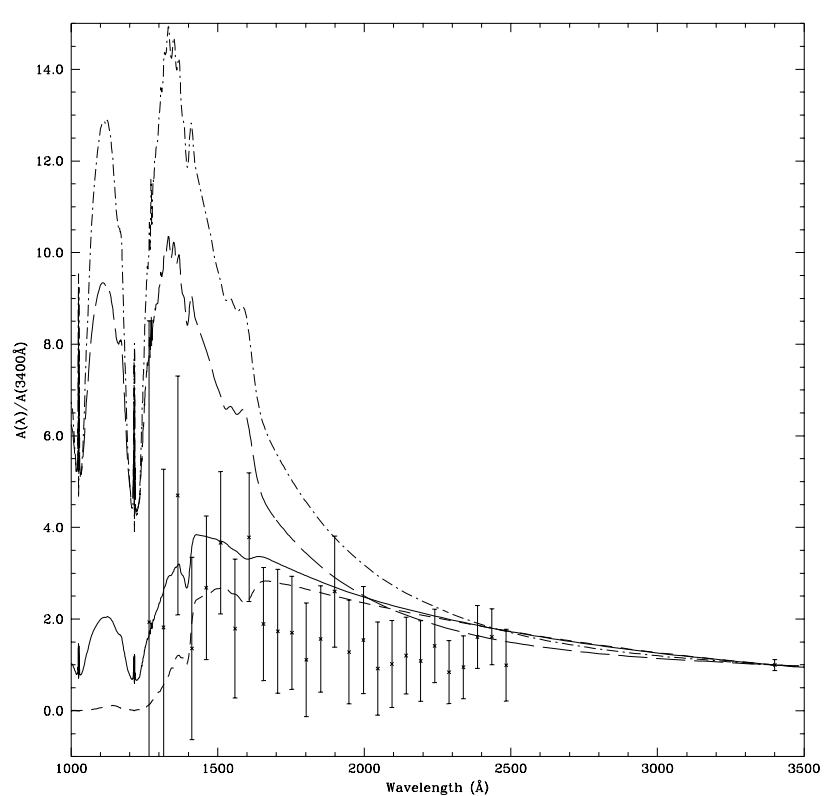

Fig. 10. Amplitude versus wavelength for $P=141.9 \mathrm{~s}$ (points). $Y_{1, m}^{2}$ (red continuous line), $\ell=0$ (blue short-dashed line), $\ell=1$ (green long-dashed line) and $\ell=2$ (yellow dotted-dashed line) are the models for $T_{\text {eff }}=12000 \mathrm{~K}$ and $\log g=8.00$. The plotted error bars are 2 sigma total and the uncertainties on the ratios had their uncertainties propagated.

spectra. However, we do detect splittings around $141 \mathrm{~s}$ and $71 \mathrm{~s}$. If we assume the observed frequencies are due to the rotational splitting of an $\ell=2$ mode, then $P_{\text {rot }} \simeq 0.7 \mathrm{~h}$, which is fast compared to the $P_{\text {rot }} \simeq 1$ day observed for other white dwarf stars, including the DAVs G 226-29, GD 385 (Kepler et al. 1995), and HS $0507+434 \mathrm{~B}$, which has a period of 1.7 day (Handler et al. 2002). In spite of the no detection by TC of any velocity variation at any frequency, indicating that the angle between the pulsations axis and the line-of-sight is $90 \mathrm{deg}$ if the pulsations are $m=0$, we detected the largest number of simultaneous pulsation of any ZZ Ceti star. The largest number of pulsations should occur for a star at the red edge, where the pulsation amplitude is the highest. As the star cools, the convection zone gets deeper, and the layer above it gets larger, allowing more frequencies to tune in. G 29-38 is an example: many frequencies present, as the other red edge pulsators. Kleinman et al. (1998) studied G 29-38, a cool DAV, and determined 19 pulsation modes for this star.

Koester et al. (1998) found line core broadening of up to $45 \mathrm{~km} \mathrm{~s}^{-1}$ for some pulsating white dwarf (ZZ Ceti) stars, compared to $4.5 \mathrm{~km} \mathrm{~s}^{-1}$ for non ZZ Cetis. We note that, even though Clemens et al. (2000) and Thompson et al. (2003) only detected velocities amplitudes up to $4.5 \mathrm{~km} \mathrm{~s}^{-1}$ in ZZ Cetis, and found similar widths at the average blue and red shifted phases, these values represent Fourier velocity amplitudes, not peak-to-peak amplitudes.

\section{Comparison of $T_{\text {eff }}$ and logg with other methods}

The measured parallax of the star is $0.056 \pm 0.003^{\prime \prime}$ (van Altena et al. 2001), and its apparent magnitude is $V=12.97 \pm 0.01$ (Dahn et al. 1976). Using these values, we calculated the

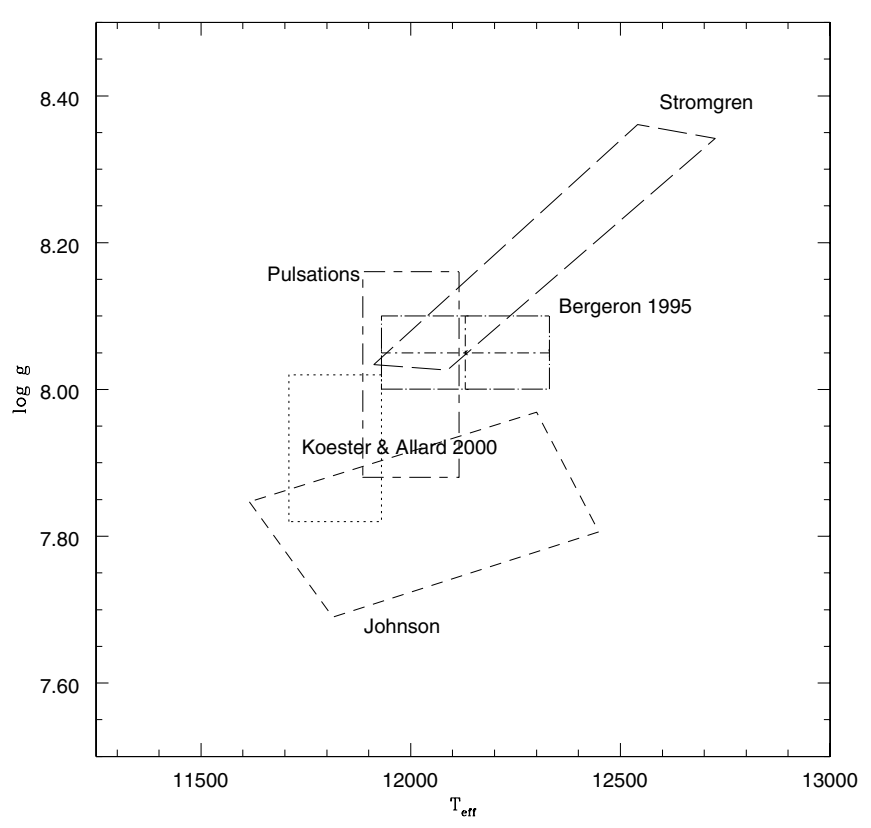

Fig. 11. Determinations of $T_{\text {eff }}$ and $\log g$ from different methods. The long-short dashed box labeled Pulsations is the result of the determination from the comparison of the amplitude in the ultraviolet with the optical. The boxes labeled Stromgren (long dashed) and Johnson (short dashed) are the determinations from color indices in comparison with Bergeron's et al. (2001) model colors. The dot-dashed box labeled Bergeron 1995 is his result from optical spectra. The dotted box labeled Koester \& Allard (2000) is their determination from ultraviolet spectrum, $V$ magnitude and parallax.

absolute magnitude $M_{V}=11.36$, and compared them with Bergeron et al.'s (2001) atmospheric models. The result defines possible combined solutions for $T_{\text {eff }}$ and $\log g$.

There are published Johnson (Dahn et al. 1988), Strömgren (Lacombe \& Fontaine 1981; Wegner 1983) and Greenstein colors (Greenstein 1984) for G 185-32, which we also compared to Bergeron's et al. (2001) model colors. For Strömgren colors, we considered the external error bars, taking into account the two measurements. For Johnson colors, there are no error bars published, so we considered that the minimum internal error is at least 0.03 . We did not use Greenstein colors, because no blue colors are available, i.e., it is not possible to determine gravity from the published colors. The effect of gravity on colors and spectra is dominant in the blue region because the hydrogen levels with $n \simeq 7$ and higher, corresponding to lines $\mathrm{H} \epsilon$ or bluer in the optical, are the ones significantly displaced by high pressure.

We also compared the time-averaged HST spectrum with Koester's theoretical spectra derived from model atmosphere, not fixing any value of $T_{\text {eff }}$ or $\log g$ as assumed by Kepler et al. (2000). In this kind of analysis we found possible ( $T_{\text {eff }}$, $\log g$ ) solutions.

In Fig. 11 we show the solutions derived by these methods, and the determination from optical spectra (Bergeron 1995). The boxes represent an error bar of $\pm 1 \sigma$. The methods of determination are based on independent data sets. If we consider probability densities with normal distributions for each method, the best solution given by the product of all 
probabilities is $T_{\text {eff }}=11960 \pm 80 \mathrm{~K}$ and $\log g=8.02 \pm$ 0.04 , corresponding to a mass of $0.617 \pm 0.024 M_{\odot}$ from Wood (1995) evolutionary models.

\section{Concluding remarks}

We conclude that the star has at least 12 pulsation modes, the ones we could attribute an $\ell$ value to. The $141.9 \mathrm{~s}$ periodicity is probably due to nonlinear effects, not a true pulsation. The 70.9 s pulsation mode has $\ell=2$, probably $k=1$. The best $T_{\text {eff }}$ and $\log g$ consistent with all independent data are $11960 \pm$ $80 \mathrm{~K}$ and $8.02 \pm 0.04$, corresponding to a mass of $0.617 \pm$ $0.024 M_{\odot}$ from Wood (1995) evolutionary models. The inclination angle of the pulsation axis in relation to the line-of-sight must be unfavorable, i.e., close to to perpendicular if the pulsations are $m=0$ or \pm 2 , and close to parallel otherwise.

Acknowledgements. We acknowledge the financial support from CNPq and NSF. Jan-Erik Solheim acknowledges professor Udo Renner, from Technishe Universität Berlin, who gave us acesss to the TUBSAT communication satellite. We acknowledge the help of the referee, Dr. Gerald Handler, for his very usefull and detailed comments, which made this paper a better one.

\section{References}

Bergeron, P., Wesemael, F., Lamontagne, R., et al. 1995, ApJ, 449, 258

Bergeron, P., Leggett, S. K., \& Ruiz, M. T. 2001, ApJS, 133, 413

Bradley, P. A. 1996, ApJ, 468, 350

Bradley, P. A. 2001, ApJ, 552, 326

Buchler, J. R., Goupil, M.-J., \& Hansen, C. J. 1997, A\&A, 321, 159

Clemens, J. C., van Kerkwijk, M. H., \& Wu, Y. 2000, MNRAS, 314, 220

Dahn, C. C., Harrington, R. S., Riepe, B., Y., et al. 1976, Publications of the US Naval Observatory Second Series, 24, 1

Dahn, C. C., Harrington, R. S., Kallarakal, V. V., et al. 1988, AJ, 95, 237

Finley, D. S., Koester, D., \& Basri, G. 1997, ApJ, 488, 375

Goldreich, P., \& Wu, Y. 1999, ApJ, 511, 904

Greenstein, J. L. 1984, ApJ, 276, 602

Handler, G., Romero-Colmenero, E., \& Montgomery, M. H. 2002, MNRAS, 335, 399
Handler, G. 2003, Baltic Astron., 12, 253

Ising, J., \& Koester, D. 2001, A\&A, 374, 116

Kanaan, A., Kepler, S. O., \& Winget, D. E. 2002, A\&A, 389, 896

Kepler, S. O. 1984, ApJ, 278, 754

Kepler, S. O. 1993, Baltic Astron., 2, 515

Kepler, S. O., Robinson, E. L., \& Nather, R. E. 1995, in Calibrating Hubble Space Telescope: Post Servicing Mission, ed. A. Koratkar, \& C. Leitherer (Baltimore: Space Telescope Science Institute), 104

Kepler, S. O., Robinson, E. L., Koester, D., et al. 2000, ApJ, 539, 379

Kepler, S. O., Nather, R. E., Winget, D. E., et al. 2003, A\&A, 401, 639

Kleinman, S. J., Nather, R. E., Winget, D. E., et al. 1998, ApJ, 495, 424

Koester, D., Dreizler, S., Weidemann, V., \& Allard, N. F. 1998, A\&A, 338,612

Koester, D., \& Allard, N. F. 2000, Baltic Astron., 9, 119

Lacombe, P., \& Fontaine, G. 1981, A\&AS, 43, 367

McGraw, J. T., Fontaine, G., Lacombe, P., et al. 1981, ApJ, 250, 349

Nather, R. E., Winget, D. E., Clemens, J. C., Hansen, C. J., \& Hine, B. P. 1990, ApJ, 361,309

Pesnell, W. D. 1985, ApJ, 292, 238

Robinson, E. L. 1979, in White Dwarf and Variable Degenerate Stars, ed. H. M. Van Horn, \& V. Weideman (Rochester, NY: University of Rochester), IAU Colloq., 53, 343

Robinson, E. L., Kepler, S. O., \& Nather, R. E. 1982, ApJ, 259, 219

Robinson, E. L., Mailloux, T. M., Zhang, E., et al. 1995, ApJ, 438, 908

Saffer, R. A., Livio, M., \& Yungelson, L. R. 1998, ApJ, 502, 394

Scargle, J. D. 1982, ApJ, 263, 835

Schwarzenberg-Czerny, A. 1999, ApJ, 516, 315

Schwarzenberg-Czerny, A. 1991, MNRAS, 253, 198

Standish, E. M. 1998, A\&A, 336, 381

Thompson, S. E., \& Clemens, J. C. 2003, in Proc. of the Asteroseismology Across the HR Diagram, ed. M. J. Thompson, M. S. Cunha, \& M. J. P. F. G. Monteiro (Dordrecht: Kluwer), 257 (TC)

Thompson, S. E., Clemens, J. C., van Kerkwijk, M. H., \& Koester, D. 2003, ApJ, 589, 921

van Altena, W. F., Lee, J. T., \& Hoffleit, E. D. 2001, VizieR Online Data Catalog, 1238

Wegner, G. 1983, AJ, 88, 109

Wu, Y. 1998, Excitation and Saturation of White Dwarf Pulsations, $\mathrm{Ph} . \mathrm{D}$. Thesis, Caltech

Wu, Y., \& Goldreich, P. 1999, ApJ, 519, 783

Wu, Y. 2001, MNRAS, 323, 248

Wu, Y., \& Goldreich, P. 2002, ApJ, 564, 1024 